\title{
Linear stability analysis of coriolis force on ferrothermohaline convection saturating an anisotropic porous medium with Soret effect
}

\author{
R. Sekar*, K. Raju, R. Vasanthakumari \\ Department of Mathematics, Pondicherry Engineering College, Puducherry - 605 014, India \\ Department of Mathematics, Pondicherry Engineering College, Puducherry - 605 014, India \\ Kasthurba College for Women, Villianur, Puducherry - 605 110, India \\ *Corresponding author E-mail: rsekar@pec.edu
}

\begin{abstract}
Instability of themocovection in a multi-component fluid has wide range of applications in ionospheric, geothermal and industries. In this analysis, the effect of rotation and vertical anisotropy on Soret-driven thermoconvective instability in a ferrofluid has been studied. The fluid layer is assumed to be horizontal and is heated from below and salted from above. In momentum equation, the effect of both substantial derivatives and coriolis terms are considered. The resulting eigen value problem is solved using Brinkman model. A linear stability analysis is used for both stationary and oscillatory instabilities for different parameters for which normal mode technique is applied. The effect of rotation tends to stabilize the system and anisotropy and Soret effects tend to destabilize the system.
\end{abstract}

Keywords: Rotation, Thermohaline convection, Anisotropic porous medium, Soret-Parameter, Ferroconvection, Ferrofluids, Multi-component fluid.

\section{Introduction}

One of the most important features of colloidal suspension of magnetic nanoparticles, known as ferrofluids, is the relative change of their viscosity with changing magnetic field. Magnetic fluid has been used in a wide variety of applications for many years by NASA in 1960s for controlling liquids in space, damping system, avionics and ball bearings, lubrications.

Numerous ferrofluids have been prepared tailor-made for requisite applications industries. One of the applications of ferromagnetic fluid is the sealing of rotating shafts and the benefit is used in various technical applications like sealing of hard disk drives, rotating X-ray tubes or rotating vacuum feed-through where reliable sealing through low friction is required. In electrical and electronic industries, ferrofluid is used to improve hi-fi characteristic loud speakers, fluid as transformer coolants and in miniaturizing inductive components, which for a long time eluded the electrical industries. The use of magnetic fluids as a heat transfer medium that may be magnetically most important branch of manufacturing. In the field of biomedicine, the application of magnetic fluid for the purpose of cancer treatment either by hyperthermia, using the change of magnetization in an air condition field to heat the tissue, or by targeting are obviously challenging possibilities.

An authoritative introduction to excellent reviews of this fascinating subject has been discussed in monograph by Rosensweig [1] and the study of the effect of magnetization yields interesting information. Usually, this magnetization is a function of magnetic field, the temperature and density of the fluid. This in presence of a gradient of magnetic field gives convection in ferromagnetic fluids which is known as ferroconvection and is similar to Bénard convection in ordinary fluids (Chandrasekhar [2]). Convective instability of a ferromagnetic fluid for a fluid layer heated from below in the presence of uniform vertical magnetic field has been considered by Finlayson [3]. Lalas and Carmi [4] studied thermoconvective instability without considering buoyancy effects.

Ferrofluids are single-magnetic-domain, two-phase three-component fluids, where the core stands for the single domain, core and carrier fluids stands for the two phases, and core, surfactant and carrier fluids stands for three component. Attempts have been made to analyze convective and conductive heat transports in strongly magnetized fluids [5]. The Soret driven ferrothermohaline convective system is a double diffusive convective system. This convective instability in multi-component [6] fluids has various applications in heat and mass transfer.

During the last four decades there has been a great deal of effort lead to by many researchers on the study of effect of rotation and anisotropy on ferroconvection [7-12] for single component fluid. The anisotropy of viscosity of a ferrofluid with 
magnetically interacting particles which are able to form structures in an applied magnetic field has been investigated by Gerch-Noritzsch et al. [13]. The effect of Coriolis force on the onset of ferromagnetic convection in a rotating horizontal ferrofluid saturated a porous layer in the pressure of a uniform vertical magnetic field studied by Shivakumara et al. [14]. Sunil et al. [15] investigated theoretically the effect of rotating ferromagnetic fluid heated from below in a porous medium in the presence of dust particles.

In double diffusive convection, the effect of rotation on a layer of a ferromagnetic fluid permeated with dust particles heated and soluted from below has been studied by Sunil et al. [16]. Sunil et al. [17] considered the double diffusive convection in magnetized ferrofluid with internal angular momentum in the presence of rotation. Malashetty et al. [18] investigated the double diffusive convection in a fluid saturated rotating sparsely packed porous layer. This investigation is used by Brinkman and thermal non-equilibrium models. Malashetty and Rajashekar Heera [19] studied the effect of rotation and anisotropy on the onset of double diffusive convection. Benano-Melly et al. [20] studied to reproduce the Soret number measurement experiments in porous media considered thermal and solutal convections. Sekar et al. [21, 22] investigated the presence of rotation on ferrothermohaline convection for two component fluid with existence and non-existence of a porous medium. Sekar et al. [23] and Vaidyanathan et al. [24] have studied the presence of coriolis effect on Soret-driven ferrothermohaline convection and extended to a porous medium for multicomponent fluid. More recently, Sekar et al. [25] studied the linear analytical study of Soret-driven ferrothermohaline convection in an anisotropic porous medium of sparse particle suspension.

In the present investigation, the treatment of rotation on Soret-driven ferrothermo convective instability of multicomponent fluid heated from below and salted from above for different range of anisotropic porous medium has been analysed for a linear stability analysis.

\section{Mathematical formulation of problem}

An infinitely spread horizontal layer of Boussinesq magnetic fluid of thickness ' $d$ ' saturating a sparsely rotating anisotropic porous medium in the presence of transverse applied magnetic field heated from below and salted from above is considered. The entire system is assumed to be rotating in the vertical direction of $z$-axis, with uniform right handed angular velocity $\mathbf{\Omega}$. The anisotropy and isotropy are considered along vertical and horizontal directions respectively. The temperature and salinity at $z=\mathrm{md} / 2$ are $T_{0} \pm(\Delta T) / 2$ and $S_{0} \mathrm{~m}(\Delta S) / 2$ respectively. Both boundaries are taken to be free and perfect conductors of heat and salt.

The momentum equation for a Brinkman model is

$$
\rho_{0}(\partial / \partial t+\mathbf{q} \cdot \nabla) \mathbf{q}=-\nabla p+\rho \mathbf{g}+\nabla \cdot(\mathbf{H B})+\mu \nabla^{2} \mathbf{q}+2 \rho_{0}(\mathbf{q} \times \mathbf{\Omega})-\frac{\mu}{k} \mathbf{q}+\frac{\rho_{0}}{2} \nabla\left(|\mathbf{\Omega} \times \mathbf{r}|^{2}\right)
$$

The continuity equation for Boussinesq fluid is

$$
\nabla \cdot \mathbf{q}=0
$$

The temperature equation for an incompressible fluid given Sekar et al. [8] is

$$
\left[\rho_{0} C_{v, H}-\mu_{o} \mathbf{H} \cdot(\partial \mathbf{M} / \partial T)_{v, H}\right](d T / d t)+\mu_{0} T(\partial \mathbf{M} / \partial T)_{v, H} \cdot(d \mathbf{H} / d t)=K_{1} \nabla^{2} T+\phi
$$

The mass flux equation is given by

$$
\rho_{0}(\partial / \partial t+\mathbf{q}) S=K_{S} \nabla^{2} S+S_{T} \nabla^{2} T
$$

where $\rho_{0}, D / D t, \mathbf{q}, t, \mathbf{H}, \mathbf{B}, p, \mathbf{g}, \mu, \Omega, k, C_{v, \mathrm{H}}, \mathbf{M}, T, K_{1}, K_{S}, S, \phi$ and $S_{T}$ are the density, substantial derivative, velocity, time, magnetic field, magnetic induction, pressure, acceleration due to gravity, dynamic viscosity (constant), angular velocity vector, permeability of the porous medium, heat capacity at constant volume and magnetic field, magnetization, temperature, thermal conductivity, concentration diffusivity, salinity, viscous dissipation factor containing second-order terms in velocity and Soret coefficient, respectively.

Further analysis has been carried out using the techniques of [3] and [21]-[25]. The magnetic equation of state is linearised about the magnetic field $H_{0}$, the average temperature $T_{0}$ and the average salinity $S_{0}$ become

$\mathbf{M}=M_{0}+\chi\left(\mathrm{H}-H_{0}\right)-K\left(T-T_{0}\right)+K_{2}\left(S-S_{0}\right)$,

where $\chi=(\partial \mathrm{M} / \partial H)_{H_{0}, T_{0}}$ is the magnetic susceptibility, $K=-(\partial \mathrm{M} / \partial T)_{H_{0}, T_{0}}$ is the pyromagnetic coefficient and $K_{2}=(\partial \mathrm{M} / \partial S)_{H_{0}, T_{0}}$ is the salinity magnetic coefficient.

The Maxwell's equations for non-conducting fluids are 
$\nabla . \mathbf{B}=0, \quad \nabla \times \mathbf{H}=0$

Also, $\mathbf{B}$ and $\mathbf{H}$ are related by

$\mathbf{B}=\mu_{0}(\mathbf{B}+\mathbf{H})$

The magnetization is depends on the magnitude of magnetic field, temperature and salinity. Using Maxwell's equation for non-conducting fluids, the magnetization is written as

$\mathbf{M}=\frac{\mathbf{H}}{H} M(H, T, S)$.

The density equation of state for a two-component fluid [23]-[25] is

$\rho=\rho_{0}\left[1-\alpha_{t}\left(T-T_{0}\right)+\alpha_{S}\left(S-S_{0}\right)\right]$

where $\alpha_{t}$ is the thermal expansion coefficient and $\alpha_{S}$ is the solute analog of $\alpha_{t}$.

The basic state is assumed to be quiescent state. Taking the components of magnetization and magnetic field in the quiescent state as $\left[0,0, M_{0}(z)\right]$ and $\left[0,0, H_{0}(z)\right]$, we obtain the following basic states quantities are

$\left.\begin{array}{l}\stackrel{\mathrm{r}}{q_{b}}=0 ; T_{b}(z)=T_{0}-\beta_{t} z ; S_{b}(z)=S_{0}-\beta_{S} z ; \quad p=p_{b} ; \rho_{b}=\rho_{0}\left(1+\alpha_{t} \beta_{t} z-\alpha_{s} \beta_{s} z\right) ; \\ H_{b}(z)=\left[H_{0}+\frac{K\left(T_{b}-T_{0}\right)}{1+\chi}-\frac{K_{2}\left(S_{b}-S_{0}\right)}{1+\chi}\right] \hat{k} ; M_{b}(z)=\left[M_{0}-\frac{K\left(T_{b}-T_{0}\right)}{1+\chi}+\frac{K_{2}\left(S_{b}-S_{0}\right)}{1+\chi}\right] \hat{k} .\end{array}\right\}$

The basic state quantities are perturbed by a small thermal disturbance. Let the components of the perturbed magnetic field and magnetization be $\left(H_{1}^{\prime}, H_{2}^{\prime}, H_{0}(z)+H_{3}^{\prime}\right)$ and $\left(M_{1}^{\prime}, M_{2}^{\prime}, M_{0}(z)+M_{3}^{\prime}\right)$, respectively.

The

perturbed temperature and salinity are taken to be $T=T_{0}-\beta_{t} z+T^{\prime}$ and $S=S_{0}-\beta_{t} z+S^{\prime}$ respectively, where primed quantities denoted the perturbation.

Using linear theory, the Eqs. (5) and (7) yields

$\left.\begin{array}{l}H_{i}^{\prime}+M_{i}^{\prime}=\left[1+\left(M_{0} / H_{0}\right)\right] H_{i}^{\prime}, \quad \text { for } i=1,2 \\ H_{3}^{\prime}+M_{3}^{\prime}=(1+\chi) H_{3}^{\prime}-K_{2} S+S_{T} K \theta-K \theta\end{array}\right\}$

where it is assumed that $K \beta_{t} d<(1+\chi) H_{0}$ and $K_{2} \beta_{S} d<<(1+\chi) H_{0}$.

Further,

$B_{i}^{\prime}=\mu_{0}\left[1+\left(M_{0} / H_{0}\right)\right] H_{i}^{\prime} \quad$ for $i=1,2$

$\left.B_{3}=\mu_{0}\left(M_{0}+H_{0}\right)+\mu_{0}(1+\chi) H_{3}^{\prime}-\mu_{0} K_{2} S+\mu_{0} S_{T} K \theta-\mu_{0} K \theta\right\}$

The Eq. (6b) implies that $\mathrm{H}^{\prime}=\nabla \varphi^{\prime}$, where $\varphi^{\prime}$ is the perturbed magnetic scalar potential. Normal mode solutions of all dynamical variables can be written as

$\left[\begin{array}{c}w \\ \theta \\ \varphi \\ S\end{array}\right]=\left[\begin{array}{l}w(z, t) \\ \theta(z, t) \\ \varphi(z, t) \\ S(z, t)\end{array}\right] \exp \left[i k_{x} x+i k_{y} y\right]$

where $k_{0}$ is the wave number is given by $k_{0}^{2}=k_{x}^{2}+k_{y}^{2}$.

The vertical component of the momentum equation can be written as

$$
\left.\begin{array}{rl}
\rho_{0} \frac{\partial}{\partial t}\left(\frac{\partial^{2}}{\partial z^{2}}-k_{0}^{2}\right) w & =\left(\frac{\mu_{0} K \beta_{t}}{1+\chi}\right)\left[(1+\chi) \frac{\partial \phi}{\partial z}-K \theta\left(1-S_{T}\right)\right] k_{0}^{2}-\rho_{0} g \alpha_{t} k_{0}^{2} \theta+\left(\frac{\mu_{0} K_{2} \beta_{S}}{1+\chi}\right)\left[(1+\chi) \frac{\partial \phi}{\partial z}+K_{2} S\right] k_{0}^{2} \\
& +\rho_{0} g \alpha_{S} k_{0}^{2} S-\left(\frac{\mu_{0} K K_{2}}{1+\chi}\right)\left[\beta_{S}\left(1-S_{T}\right) \theta-\beta_{t} S\right] k_{0}^{2}+\mu\left(\frac{\partial^{2}}{\partial z^{2}}-k_{0}^{2}\right)^{2} w+\frac{\mu}{k^{\prime}} k_{0}^{2} w-\frac{\mu}{k_{1}} \frac{\partial^{2} w}{\partial z^{2}}-2 \rho_{0} \Omega \frac{\partial \xi}{\partial z}
\end{array}\right\}
$$

where $\xi=\frac{\partial v}{\partial x}-\frac{\partial u}{\partial y}$ is the vertical component of vorticity.

The vertical component of vorticity equation can be written as 
$\left(\rho_{0} \frac{\partial}{\partial t}+\frac{\mu}{k_{1}}\right) \xi=\mu_{0}\left(\frac{\partial^{2}}{\partial z^{2}}-k_{0}^{2}\right) \xi+2 \rho_{0} \Omega \frac{\partial w}{\partial z}$

The modified Fourier heat conduction equation is

$\rho_{0} C_{v, H} \frac{\partial \theta}{\partial t}-\mu_{0} K T_{0} \frac{\partial}{\partial t}\left(\frac{\partial \phi}{\partial z}\right)=K_{1}\left(\frac{\partial^{2}}{\partial z}-k_{0}^{2}\right) \theta+\left[\rho_{0} c \beta_{t}-\left(\frac{\mu_{0} K^{2} T_{0}^{2} \beta_{t}}{1+\chi}\right)+\left(\frac{\mu_{0} K K_{2} T_{0} \beta_{S}}{1+\chi}\right)\right] w$

where $\rho_{0} C=\rho_{0} C_{v, H}+\rho_{0} K H_{0}$.

The Salinity equation is

$$
\frac{\partial S}{\partial t}+\beta_{S} w=K_{S}\left(\frac{\partial^{2}}{\partial z^{2}}-k_{0}^{2}\right) S+S_{T}\left(\frac{\partial^{2}}{\partial z^{2}}-k_{0}^{2}\right) \theta
$$

Using the analysis similar to Sekar et al. [23], one gets

$(1+\chi) \frac{\partial^{2} \phi}{\partial z^{2}}-\left(1+\frac{M_{0}}{H_{0}}\right) k_{0}^{2} \phi-K \frac{\partial \theta}{\partial z}+K_{2} \frac{\partial S}{\partial z}+S_{T} K \frac{\partial \theta}{\partial z}=0$

Following the analysis of [3] and [21]-[25], the equations in non-dimensional form can be written using

$$
\begin{aligned}
& w^{*}=\frac{w d}{v}, t^{*}=\frac{v t}{d^{2}}, T^{*}=\left(\frac{K_{1} a R^{1 / 2}}{\rho_{0} C_{v, H} \beta_{t} v d}\right) \theta, \phi^{*}=\left(\frac{(1+\chi) K_{1} a R^{1 / 2}}{\rho_{0} C_{v, H} K \beta_{t} v d^{2}}\right) \phi, z^{*}=\frac{z}{d}, \\
& \left.\begin{array}{l}
a=k_{0} d, \quad D=\frac{\partial}{\partial z^{*}}, S^{*}=\left(\frac{K_{S} a R_{S}^{1 / 2}}{\rho_{0} C_{v, H} \beta_{S} v d}\right) S, \quad v=\frac{\mu}{\rho_{0}}, k_{1}^{*}=\frac{k_{1}}{d^{2}}, k^{*}=\frac{k_{2}}{d^{2}}, \xi^{*}=\frac{\xi d^{2}}{v^{2}}
\end{array}\right\}
\end{aligned}
$$

Then the Eqs. (14)-(18) become

$$
\begin{aligned}
& \left.\begin{array}{rl}
\frac{\partial}{\partial t^{*}}\left(D^{2}-a^{2}\right) w^{*}= & a R^{1 / 2}\left[M_{1} D \phi^{*}-\left(1+M_{1}\left(1-S_{T}\right) T^{*}\right)\right]+a R^{1 / 2} M_{1} M_{5} D \phi^{*}-a R^{1 / 2} M_{1} M_{5}\left(1-S_{T}\right) T^{*} \\
& +\left(D^{2}-a^{2}\right)^{2} w^{*}+a R_{S}^{1 / 2}\left[1+M_{4}+\frac{M_{4}}{M_{5}}\right] S^{*}-\left(\frac{D^{2}}{k_{1}^{*}}-\frac{a^{2}}{k^{*}}\right) w^{*}-\left(T_{a}\right)^{1 / 2} D \xi^{*},
\end{array}\right\} \\
& \left(\frac{\partial}{\partial t}+\frac{1}{k_{1}^{*}}\right) \xi^{*}=\left(D^{2}-a^{2}\right) \xi^{*}+\left(T_{a}\right)^{1 / 2} D w^{*}, \\
& P_{r}\left[\frac{\partial T^{*}}{\partial t^{*}}-M_{2} \frac{\partial}{\partial t^{*}}\left(D \phi^{*}\right)\right]=\left(D^{2}-a^{2}\right) T^{*}+a R^{1 / 2}\left(1-M_{2}-M_{2} M_{5}\right) w^{*}, \\
& P_{r} \frac{\partial S *}{\partial t^{*}}=\tau\left(D^{2}-a^{2}\right) S^{*}-a R_{S}^{1 / 2} M_{6} w^{*}+S_{T}\left(\frac{M_{5}}{M_{6}}\right)\left(\frac{R}{R_{S}}\right)^{1 / 2}\left(D^{2}-a^{2}\right) T^{*},
\end{aligned}
$$

and

$$
D^{2} \phi^{*}-M_{3} a^{2} \phi^{*}-\left(1-S_{T}\right) D T *+\frac{M_{5}}{M_{6}}\left(\frac{R}{R_{S}}\right)^{1 / 2} D S^{*}=0,
$$

where the non-dimensional parameters used are

$$
\begin{aligned}
& M_{1}=\frac{\mu_{0} K^{2} \beta_{t}}{(1+\chi) \rho_{0} g \alpha_{t}}, M_{2}=\frac{\mu_{0} K^{2} T}{(1+\chi) \rho_{0} C_{v, H}}, M_{3}=\frac{1+M_{0} / H_{0}}{(1+\chi)}, M_{4}=\frac{\mu_{0} K^{2} \beta_{s}}{(1+\chi) \rho_{0} g \alpha_{s}}, M_{5}=\frac{K_{2} \beta_{s}}{K \beta_{t}}, M_{6}=\frac{K_{S}}{K_{1}}, \tau=\rho_{0} C_{v, H}\left(\frac{K_{S}}{K_{1}}\right), \\
& P_{r}=\frac{\rho_{0} C_{v, H}}{K_{1}}, R_{S}=\frac{\rho_{0} C_{v, H} \beta_{s} \alpha_{s} g d^{4}}{v K_{S}}, R=\frac{\rho_{0} C_{v, H} \beta_{t} \alpha_{t} g d^{4}}{v K_{1}}, \quad\left(T_{a}\right)^{1 / 2}=\frac{2 \Omega d^{2}}{v},
\end{aligned}
$$


where $R_{S}$ is the salinity Rayleigh number, $R$ is the thermal Rayleigh number, $P_{r}$ is the Prandtl number and other parameters described non-dimensional parameters.

\section{Free boundaries solutions}

The boundary conditions on velocity, temperature, salinity and angular momentum are $w^{*}=D^{2} w^{*}=T^{*}=D \phi^{*}=S^{*}=\xi^{*}=D \xi^{*}=0$ at $z^{*}= \pm 1 / 2$.

The exact solutions satisfying above Eq. (26) are

$$
\left.\begin{array}{l}
w^{*}=A_{1} e^{\sigma t^{*}} \cos \pi z^{*}, \quad T^{*}=B_{1} e^{\sigma t^{*}} \cos \pi z^{*}, \quad S^{*}=C_{1} e^{\sigma t^{*}} \cos \pi z^{*} \\
D \phi^{*}=E_{1} e^{\sigma t^{*}} \cos \pi z^{*}, \quad \phi^{*}=\frac{E_{1}}{\pi} \sin \pi z^{*}
\end{array}\right\}
$$

where $A_{1}, B_{1}, C_{1}$ and $E_{1}$ are constants. These functions substituted in the set of Eqs. (20) - (24) gives the following four linear homogeneous algebraic equations in the constant $A_{1}, B_{1}, C_{1}$ and $E_{1}$ are obtained upon $k^{*^{*}}=\varepsilon k_{1}^{*}$ and removing the asterisks for our convenience, where $\varepsilon$ is non-dimensional parameter governing anisotropy leads to

$$
\begin{aligned}
& \left.\left[\sigma\left(\pi^{2}+a^{2}\right)+\left(\pi^{2}+a^{2}\right)^{2}+\left(\pi^{2} \varepsilon+a^{2}\right) / k_{1} \varepsilon+\left(T_{a} \pi^{2} /\left(\sigma+k_{1}+\pi^{2}+a^{2}\right)\right)\right] A_{1}-a R^{1 / 2}\left[1+M_{1}\left(1-S_{T}\right)+M_{1} M_{5}\left(1-S_{T}\right)\right] B_{1}\right\} \\
& \left.+a R_{S}^{1 / 2}\left(1+M_{4}+M_{4} M_{5}^{-1}\right) C_{1}+a R^{1 / 2} M_{1}\left(1+M_{5}\right) E_{1}=0\right\} \\
& a R^{1 / 2}\left(1-M_{2}-M_{2} M_{5}\right) A_{1}-\left(\pi^{2}+a^{2}+P_{r} \sigma\right) B_{1}+P_{r} \sigma M_{2} E_{1}=0, \\
& a R_{S}^{1 / 2} M_{6} A_{1}+S_{T}\left(M_{5} / M_{6}\right)\left(R_{S} / R\right)^{1 / 2}\left(\pi^{2}+a^{2}\right) B_{1}+\left[\tau\left(\pi^{2}+a^{2}\right)+\sigma P_{r}\right] C_{1}=0, \\
& -R_{S}^{1 / 2} \pi^{2}\left(1-S_{T}\right) B_{1}+R^{1 / 2} \pi^{2} M_{5} M_{6}^{-1} C_{1}+R_{S}^{1 / 2}\left(\pi^{2}+a^{2} M_{3}\right) E_{1}=0 .
\end{aligned}
$$

The determinant of the co-efficient of $A_{1}, B_{1}, C_{1}$ and $E_{1}$ in Eqs. (28)-(31) must vanish for the existence of non-trivial eigen functions. The techniques and analysis of [3] and [7]-[12] (28)-(31) have been adopted to obtain $U \sigma^{4}+V \sigma^{3}+W \sigma^{2}+X \sigma+Y=0$

where

$$
\begin{aligned}
& U=c_{1} c_{4} P_{r}^{2}+c_{1} P_{r}^{2} M_{2} \pi^{2}\left(1-S_{T}\right) \\
& V=c_{1}^{2} c_{4} P_{r}\left[(1+\tau)+P_{r}\left(e_{2}+c_{1} e_{1}\right)\right]+c_{1}^{2} P_{r} M_{2} S_{T}\left(M_{5} / M_{6}\right)^{2} \pi^{2} \\
& +\left(\tau c_{1}^{2}+\left(c_{1}^{2}+e_{2}+c_{1} e_{1}\right) P_{r}\right) P_{r} M_{2}\left(1-S_{T}\right) \pi^{2}-a\left(1+c_{2}\left(1-S_{T}\right)\right) P_{r}^{2} M_{2}\left(1-S_{T}\right) \\
& W=c_{4}\left(c_{1}^{3} \tau+\left(c_{1}\left(e_{2}+c_{1}\right)+e_{1}\right)\left(P_{r} c_{1}(1+\tau)\right)+P_{r}^{2}\left(e_{2}\left(c_{1}^{2}+e_{1}\right)+T_{a} \pi^{2}\right)-a^{2} R P_{r}\left(1+c_{2}\left(1-S_{T}\right)\right)\right) \\
& +\left(+a^{2} R P_{r} \pi^{2} c_{2}\left(\left(1-S_{T}\right)+M_{5}\right)-a^{2} R_{s} P_{r} c_{4} c_{3} M_{6}\right)+\left(c_{1}^{2}+e_{2}+c_{1} e_{1}\right) c_{1} P_{r} M_{2} S_{T}\left(M_{5} / M_{6}\right)^{2} \pi^{2} \\
& +\tau c_{1} P_{r} M_{2}\left(1-S_{T}\right) \pi^{2}-a\left(1+c_{2}\left(1-S_{T}\right)\right)\left(\tau c_{1}+P_{r} e_{2}\right) P_{r} M_{2}+a^{2} c_{3} P_{r} M_{2} M_{6}\left(1-S_{T}\right) \pi^{2} \\
& +a^{2} R\left(c_{4} c_{5}\left(1+c_{2}\left(1-S_{T}\right)\right) P_{r}-c_{2}\left(1-S_{T}\right) P_{r}\right) \\
& X=c_{4}\left[P_{r} c_{1}(1+\tau)\left(T_{a} \pi^{2}+e_{2}\left(c_{1}^{2}+e_{1}\right)\right)+c_{4} c_{1}^{2} \tau\left(\left(e_{2}+c_{1}\right) c_{1}^{2}+e_{1}\right)-a^{2} R\left(1+c_{2} c_{4}\right)\left(\tau c_{1} P_{r} e_{2}\right)\right. \\
& \left.-a^{2} R_{s} S_{T} c_{1} c_{3}\left(M_{5} / M_{6}\right)-a^{2} R_{s} c_{3} M_{6}\left(c_{1}+e_{2} P_{r}\right)\right] \\
& +c_{2} a^{2} R\left[\pi^{2}\left(\left(1-S_{T}\right)\left(\tau c_{1}+P_{r} e_{2}\right)+M_{5}\left(P_{r} e_{2}+c_{1}\right)\right)-S_{T} c_{1} \pi^{2}\left(M_{5}^{2} / M_{6}^{2}\right)\right] \\
& +T_{a} \pi^{4} P_{r} M_{2} S_{T} c_{1}\left(M_{5}^{2} / M_{6}^{2}\right)-a\left(1+c_{2}\left(1-S_{T}\right)\right) P_{r} M_{2} c_{4} e_{2} c_{1} \tau+a^{2} c_{3} P_{r} M_{2} M_{6}\left(1-S_{T}\right) e_{2} \pi^{2} \\
& +a^{2} R c_{4} c_{5}\left(1+c_{2}\left(1-S_{T}\right)\right)\left(\tau c_{1}+P_{r} e_{2}\right)-a^{2} R_{S} S_{T} c_{1} c_{3} c_{4} c_{5}\left(M_{5} / M_{6}\right) \\
& -a^{2} R S_{T} \pi^{2} c_{1} c_{2} c_{5}\left(M_{5}^{2} / M_{6}^{2}\right)-a^{2} R\left(1-S_{T}\right) \pi^{2} c_{2} c_{5}\left(\tau c_{1}+P_{r} e_{2}\right) \\
& Y=c_{4}\left\{\left[\tau c_{1}^{2} e_{2}\left(c_{1}^{2}+e_{1}\right)+T_{a} \pi^{2}\right]-a^{2} R \tau c_{1} e_{2}\left(1+\left(1-S_{T}\right) c_{2}\right) c_{5}-a^{2} R_{s} c_{1} e_{2}\left[S_{T}\left(M_{5} / M_{6}\right) c_{5}+M_{6}\right]\right\} \\
& +a^{2} R c_{1} c_{2} e_{2} \pi^{2}\left\{S_{T}\left(\left(M_{5}^{2} / M_{6}^{2}\right)+\tau\left(1-S_{T}\right)\right) c_{5}+M_{5}\right\} .
\end{aligned}
$$


where

$c_{1}=\pi^{2}+a^{2}, \quad c_{2}=M_{1}\left(1+M_{5}\right), \quad c_{3}=1+M_{4}+\left(M_{4} / M_{5}\right), \quad c_{4}=\pi^{2}+a^{2} M_{3}$, $c_{5}=1-M_{2}-M_{2} M_{5}, \quad e_{1}=\frac{\varepsilon \pi^{2}+a^{2}}{\varepsilon k_{1}}$ and $e_{2}=\frac{1}{k_{1}}+c_{1}$.

\section{The case of stationary convection}

Substitution of a factor $\sigma=i \sigma_{1}$ in Eq. (32) gives the marginal state of convection. If $\sigma_{1}$ is non-zero and real, then it gives the condition for oscillatory instability. If oscillatory instability exists, the angular frequency factor $\sigma=i \sigma_{1}$. Since $U, V, W, X$ and $Y$ are real, the Eq. (32) could be satisfied for $\sigma=i \sigma_{1}$, if and only if $\sigma=0$.

For $\sigma_{1}=0$ Rayleigh number for stationary mode is calculated from the analysis [3] and [7]-[12] of the eigen value equation and which is simplified to

$R_{s c}=\frac{N_{r}}{D_{r}}$

where

$$
\begin{array}{r}
N_{r}=\left(\pi^{2}+a^{2}\right)\left[\left(\pi^{2}+a^{2}\right)^{2}+\left(\pi^{2} \varepsilon+a^{2}\right) / \varepsilon k_{1}+\left(T_{a} \pi^{2} /\left(\left(1 / k_{1}\right)+\pi^{2}+a^{2}\right)\right)\right] \\
-a^{2} R_{s}\left(1+M_{4}+M_{4} M_{5}^{-1}\right)\left(S_{T} M_{5} M_{6}^{-1}\left(1-M_{2}-M_{2} M_{5}\right)+M_{6}\right) \tau^{-1}
\end{array}
$$

and

$$
\begin{aligned}
D_{r}= & a^{2}\left(1+\left(1-S_{T}\right) M_{1}\left(1+M_{5}\right)\left(1-M_{2}-M_{2} M_{5}\right)\right) \\
& -\left(\frac{M_{1}\left(1+M_{5}\right) \pi^{2}}{\pi^{2}+a^{2} M_{3}}\right)\left[\left(1-M_{2}-M_{2} M_{5}\right)\left(S_{T}\left(M_{5} / M_{6}\right)^{2} \tau^{-1}+\left(1-S_{T}\right)\right)+M_{5} \tau^{-1}\right]
\end{aligned}
$$

When $k_{1} \rightarrow \infty$ this tends to the critical Rayleigh number obtained by Vaidyanathan et al. [23] for multi-component fluid. This leads to the critical Rayleigh number obtained by Sekar et al. [25] for multi-component fluid as $T_{a} \rightarrow 0$. When the salinity Rayleigh number $R_{S}$ taken to be zero, this tends to the critical Rayleigh number obtained by Sekar et al. [7] for single component fluid. When the magnetization parameter $M_{l}=0$, the classical Rayleigh problem for bouncy-induced convection is obtained [2]. When all the magnetic parameters $M_{l}-M_{6}$ vanishes, this reduces to double diffusive convection [26]. When $M_{1}$ is very large, the critical magnetic thermal Rayleigh number $N_{s c}=M_{1} R_{s c}$ for stationary mode could be simplified as

$$
N_{c}=R_{s c} M_{1}=\frac{N_{r}}{D_{r}}
$$

where

$$
\begin{array}{r}
N_{r}=\left(\pi^{2}+a^{2}\right)\left[\left(\pi^{2}+a^{2}\right)^{2}+\left(\pi^{2} \varepsilon+a^{2}\right) / \varepsilon k_{1}+\left(T_{a} \pi^{2} /\left(\left(1 / k_{1}\right)+\pi^{2}+a^{2}\right)\right)\right] \\
-a^{2} R_{s}\left(1+M_{4}+M_{4} M_{5}^{-1}\right)\left(S_{T} M_{5} M_{6}^{-1}\left(1-M_{2}-M_{2} M_{5}\right)+M_{6}\right) \tau^{-1}
\end{array}
$$

and

$$
\begin{aligned}
D_{r}= & a^{2}\left(1+M_{5}\right)\left(1-S_{T}\right)\left(1-M_{2}-M_{2} M_{5}\right) \\
& -\left(\frac{\pi^{2}}{\pi^{2}+a^{2} M_{3}}\right)\left[\left(\left(1-M_{2}-M_{2} M_{5}\right)\left(S_{T}\left(M_{5} / M_{6}\right)^{2} \tau^{-1}+\left(1-S_{T}\right)\right)+M_{5} \tau^{-1}\right)\right] .
\end{aligned}
$$

Which express the critical magnetic thermal Rayleigh number $N_{c}$ is a function of critical wave number $a$, medium permeability $k_{l}$, anisotropic parameter $\varepsilon$, Taylor number $T_{a}$, salinity Rayleigh number $R_{S}$, Soret coefficient $S_{T}$, ratio of mass transport to the heat transport $\tau$, ratio of thermal flux due to magnetization to magnetic flux $M_{2}$, non-buoyancy magnetization parameter $M_{3}$, ratio of magnetic forces due to salinity fluctuation to buoyant forces $M_{4}$, ratio of salinity effect on magnetic field to pyromagnetic coefficient $M_{5}$ and ratio of mass diffusivity to thermal conductivity $M_{6}$. 


\section{The case of oscillatory mode}

Use of the factor $\sigma=i \sigma_{1}$ in Eq. (32), one gets the real value of Rayleigh number by following of [3] and [7]-[12] because the Rayleigh number is not a complex number, that is $I m R_{o c}=0$. Implies that $R_{o c}$ is a real number. Therefore the magnetic thermal Rayleigh number is calculated as

$R_{o c}=\frac{A C+B E}{A+B}$,

where

$$
\begin{aligned}
A=Y_{2} & -W_{2} \sigma_{1}^{2}, \quad B=X_{2} \sigma_{1}, \quad C=-U \sigma_{1}^{4}+W_{1} \sigma_{1}^{2}-Y_{1}, \quad E=\sigma_{1}^{3}-X_{1} \sigma_{1}, \\
W_{1}= & c_{1} P_{r}^{2}\left[e_{2}\left(c_{1}^{2}+e_{1}\right)+T_{a} \pi^{2}\right]+c_{4}\left[c_{1}^{3} \tau+\left(c_{1}\left(e_{2}+c_{1}\right)+e_{1}\right) P_{r} c_{1}(1+\tau)\right]-a^{2} R_{s} c_{3} c_{4} P_{r} M_{6} \\
& +\left(c_{1}^{2}+e_{2}+c_{1} e_{1}\right) c_{1} P_{r} M_{2} S_{T}\left(M_{5} / M_{6}\right)^{2} \pi^{2}+\tau c_{1} P_{r} M_{2}\left(1-S_{T}\right) \pi^{2} \\
& -a\left(1+c_{2}\left(1-S_{T}\right)\right)\left(\tau c_{1}+P_{r} e_{2}\right) P_{r} M_{2}+a^{2} c_{3} P_{r} M_{2} M_{6}\left(1-S_{T}\right) \pi^{2} \\
W_{2}= & a^{2} R P_{r}\left[\pi^{2} c_{1}\left(\left(1-S_{T}\right)+M_{5}\right)-c_{4}\left(1+c_{2}\left(1-S_{T}\right)\right)\right]+a^{2} R\left(c_{4} c_{5}\left(1+c_{2}\left(1-S_{T}\right)\right) P_{r}-c_{2}\left(1-S_{T}\right) P_{r}\right) \\
X_{1}= & c_{4}\left(P_{r} c_{1}(1+\tau)\left(T_{a} \pi^{2}+e_{2}\left(c_{1}^{2}+e_{1}\right)\right)+c_{1}^{2} \tau\left(\left(e_{2}+c_{1}\right) c_{1}^{2}+e_{1}\right)-a^{2} R c_{4}\left(1+c_{2} c_{4}\right)\left(\tau c_{1}+P_{r} c_{2}\right)\right) \\
& +T_{a} \pi^{4} P_{r} M_{2} S_{T} c_{1}\left(M_{5}^{2} / M_{6}^{2}\right)-a\left(1+c_{2}\left(1-S_{T}\right)\right) P_{r} M_{2} c_{4} e_{2} c_{1} \tau+a^{2} c_{3} P_{r} M_{2} M_{6}\left(1-S_{T}\right) e_{2} \pi^{2} \\
& -a^{2} R_{s} S_{T} c_{1} c_{3} c_{4} c_{5}\left(M_{5} / M_{6}\right) \\
X_{2}= & a^{2} R c_{2} \pi^{2}\left(\left(1-S_{T}\right)\left(\tau c_{1}+P_{r} e_{2}\right)+M_{5}\left(P_{r} e_{2}+c_{1}\right)+c_{1} S_{T}\left(M_{5}^{2} / M_{6}^{2}\right)\right)-a^{2} R c_{4}\left(1+c_{2} c_{4}\right)\left(\tau c_{1}+P_{r} c_{2}\right) \\
& +a^{2} R c_{4} c_{5}\left(1+c_{2}\left(1-S_{T}\right)\right)\left(\tau c_{1}+P_{r} e_{2}\right)-a^{2} R S_{T} \pi^{2} c_{1} c_{2} c_{5}\left(M_{5}^{2} / M_{6}^{2}\right) \\
& -a^{2} R\left(1-S_{T}\right) \pi^{2} c_{2} c_{5}\left(\tau c_{1}+P_{r} e_{2}\right) \\
Y_{1}= & c_{4}\left[\tau c_{1}^{2}\left(c_{4}\left(c_{1}^{2}+e_{1}\right)\right)+T_{a} \pi^{2}\right]-a^{2} R_{s} c_{1} e_{2}\left(S_{T}\left(M_{5} / M_{6}\right) c_{5}+M_{6}\right) \\
\text { and } & \\
Y_{2}= & a^{2} R c_{1}\left\{c_{2} e_{2}\left(\left(S_{T}\left(M_{5}^{2} / M_{6}^{2}\right)+\tau\left(1-S_{T}\right)\right) c_{5}+M_{5}\right)-\tau e_{2}\left(1+\left(1-S_{T}\right) c_{2} c_{5}\right)\right\} .
\end{aligned}
$$

Also $X_{1}, Y_{1}, W_{1}$ are the terms of $X, Y, W$ non containing $R$ and $X_{2}, Y_{2}, W_{2}$ are the terms containing $R$.

$$
\sigma_{1}^{2}=\frac{-B_{2} \pm \sqrt{B_{2}^{2}-4 A_{2} C_{2}}}{2 A_{2}} \text {. }
$$

where

$$
A_{2}=X_{2} U-V W_{2}, \quad B_{2}=U Y_{2}+X_{1} W_{2}-X_{2} W_{1} \text { and } C_{2}=X_{2} Y_{1}-X_{1} Y_{2}
$$

\section{Results and discussion}

Brinkman model is made on the effect of linearity on rotation and anisotropy of Soret-driven thermoconvective instability in a ferrofluid with uniform angular velocity heated from below and salted from above has been analyzed. A linear stability analysis is carried out as perturbations are small and normal mode technique is applied. The conditions for both stationary and oscillatory modes have been calculated. The range of Taylor number $T_{a}$ is chosen to study the implication of effect of rotation from a very small range to mediocre high range. 


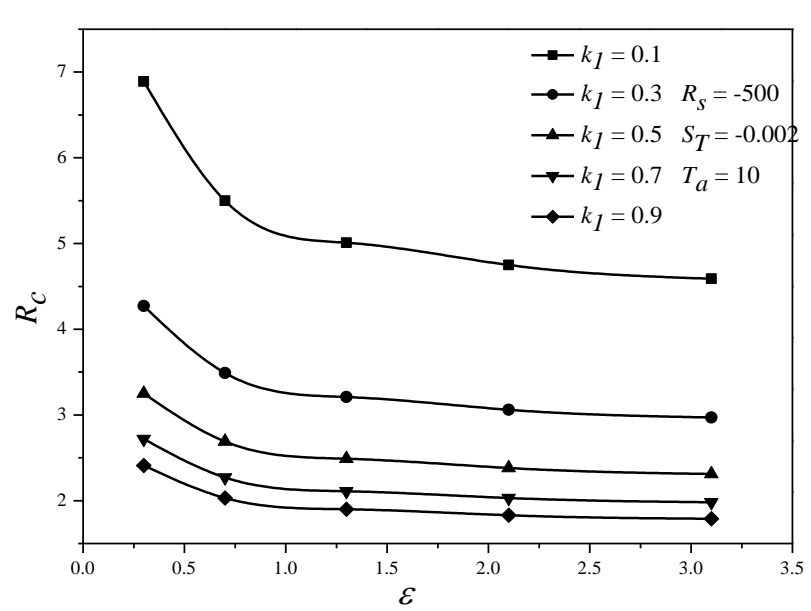

(a)

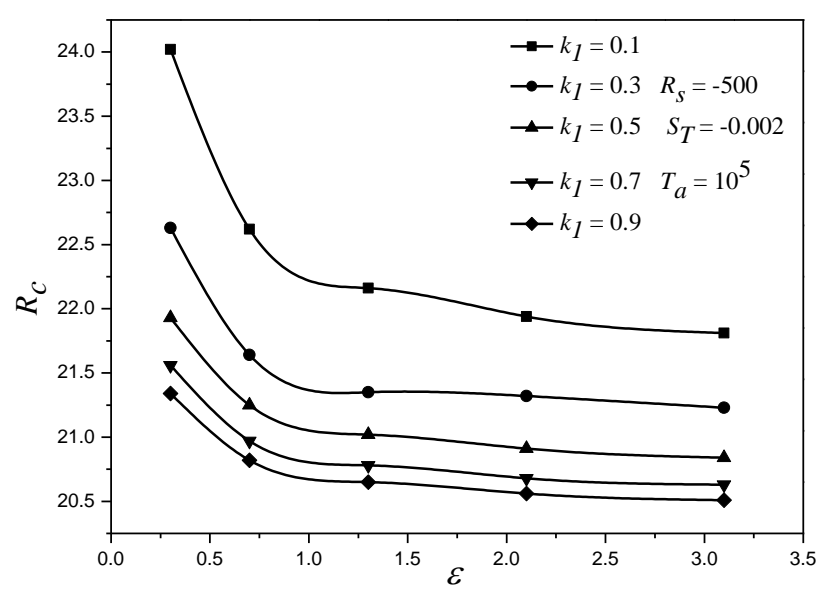

(b)

Fig. 1: (a) Variation of $R_{c}$ versus $\varepsilon$ of stationary instability for different $k_{1}, R_{S}=-500, S_{T}=-0.002$ and $T_{a}=10$.

(b) Variation of $R_{c}$ versus $\varepsilon$ of oscillatory instability for different $k_{1}, R_{s}=-500, S_{T}=-0.002$ and $T_{a}=10^{5}$.

The Taylor number $T_{a}$, which decides the effect of coriolis force, is allowed to vary from 10 to $10^{8}[23,24]$ and $T_{a}$ has defined by effect of rotation. The Prandtl number $P_{r}$ is assumed to be 0.01 [23]-[25]. The buoyancy magnetization $M_{1}$ is taken to be 1000. The value of $M_{2}$ is assumed to be zero [7]-[12] for these types of fluids. The non-buoyancy magnetization parameter $M_{3}$ is allowed to vary from 5 to 25 , because this parameter cannot take a value less than one [25]. The range of permeability of the porous medium $k_{1}$ is varied from 0.1 to 0.9 [23]-[25] and the range of an anisotropic parameter $\varepsilon$ is varied from 0.3 to 3.1 [25]. The ratio of mass transport to the heat transport $\tau$ is taken as $0.03(0.02) 0.11$ [25]. The salinity Rayleigh number $R_{\mathrm{S}}$ is taken values from -500 to 500 and Soret parameter $S_{\mathrm{T}}$ is varied from -0.002 to 0.002 and the magnetization parameters $M_{4}, M_{5}$ and $M_{6}$ are assumed to be 0.1 [23]-[25]. This system has been found to stabilize through stationary mode for $T_{a}=10$ and 100 . When $T_{a} \geq 10^{3}$, the system has stabilized through oscillatory mode.

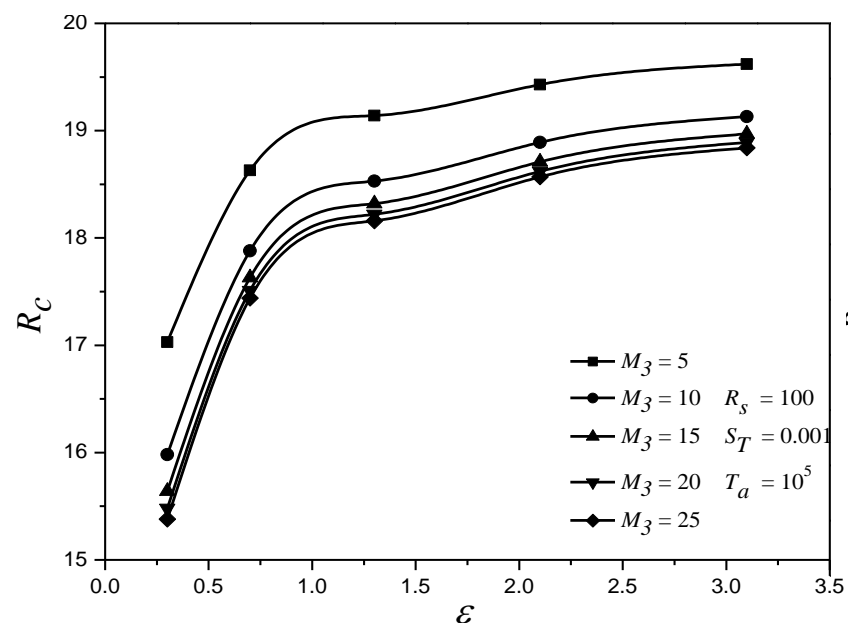

(a)

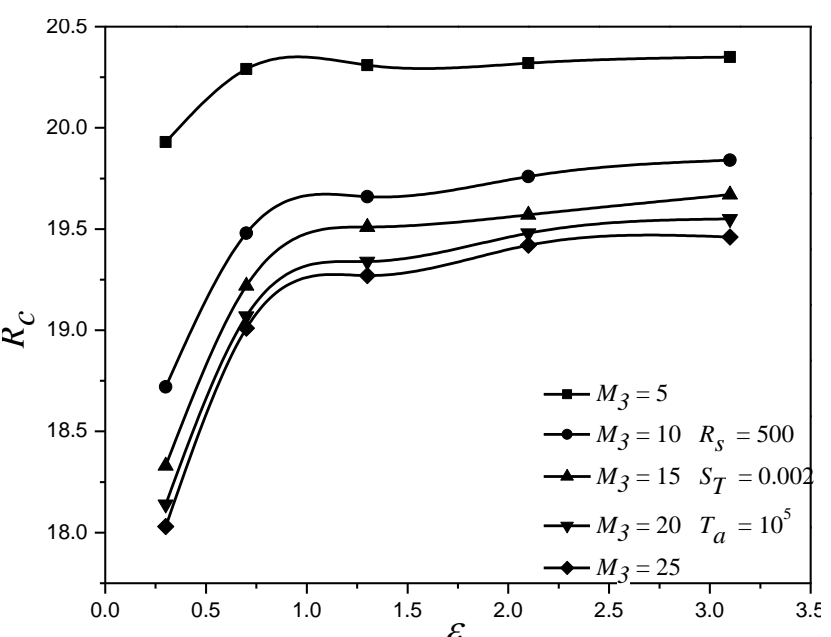

(b)

Fig. 2. (a) Variation of $R_{c}$ versus $\varepsilon$ of oscillatory instability for different $M_{3}, R_{s}=100, S_{T}=0.001$ and $T_{a}=10^{5}$.

(b) Variation of $R_{c}$ versus $\varepsilon$ of oscillatory instability for different $M_{3}, R_{s}=500, S_{T}=0.002$ and $T_{a}=10^{5}$.

From Fig. 1. (a), the cell shape and magnetic Rayleigh number $R_{c}$ with respect to the anisotropy parameter $\varepsilon$, indicate that the system destabilizes as the anisotropic parameter $\varepsilon$ increases. This is indicated by a decrease in $R_{c}$. Also as the permeability of the porous medium $k_{1}$ increases from 0.1 to 0.9 , there is a fall in the values of $R_{c}$. Thus, the large permeability is found to induce instability through the stationary mode. This is because, as the pore size increases the fluid flow takes place easily. 
It is observed from Fig. 1. (b) that permeability of the porous medium and anisotropic effect have a destabilizing behavior of the system and it gives the above onset of instability for oscillatory mode. Fig. 2. (a) gives the variation of $R_{\mathrm{c}}$ versus $\varepsilon$ for different values of magnetization parameter $M_{3}, S_{\mathrm{T}}=0.001, R_{\mathrm{s}}=100$ and $T_{a}=10^{5}$ for oscillatory mode as discussed earlier. It is clear that as the critical thermal Rayleigh number $R_{\mathrm{c}}$ increases, anisotropic parameter $\varepsilon$ increases. This tends to stabilize the system because the effect of rotation is much pronounced. Here a cell shape is evident to witness as $M_{3}=5$. But the other values of magnetization parameter $M_{3}$ varied from 10 to 25 are uniform cell shapes with thickness of the gap. However this system tends to stabilize.

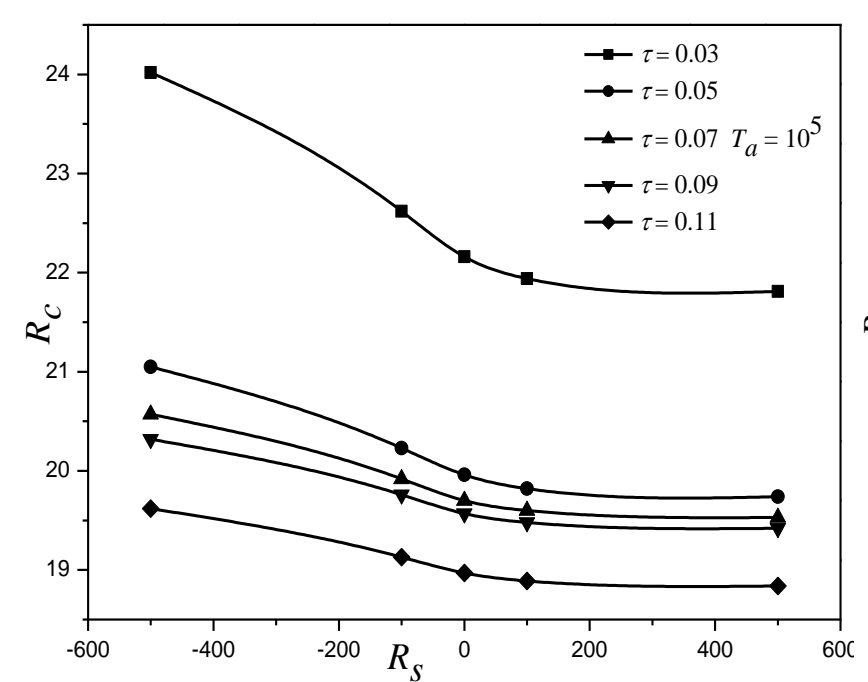

(a)

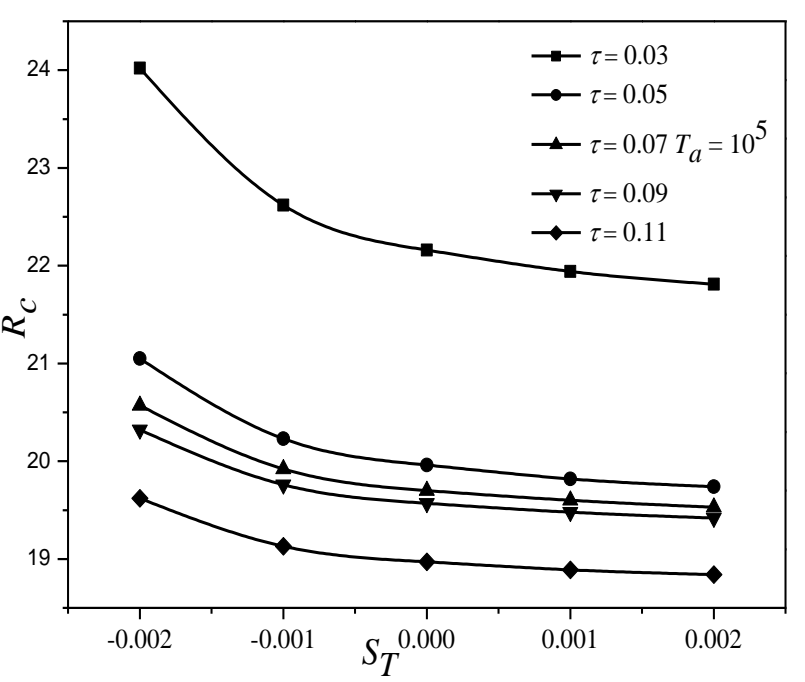

(b)

Fig. 3. (a) Variation of $R_{c}$ versus $R_{S}$ of oscillatory instability for different $\tau$ when $T_{a}=10^{5}$. (b) Variation of $R_{c}$ versus $S_{T}$ of oscillatory instability for different $\tau$ when $T_{a}=10^{5}$.

In Fig. 2. (b), the variation of $R_{\mathrm{c}}$ versus $\varepsilon$ for various values of $M_{3}, S_{\mathrm{T}}=0.002, R_{\mathrm{s}}=500$ and $T_{a}=10^{5}$ for oscillatory mode. It is seen that as critical Rayleigh number $R_{\mathrm{c}}$ increases, anisotropic parameter $\varepsilon$ increases. This tends to stabilize the behavior. Moreover, the positive range of Soret coefficient $S_{\mathrm{T}}$ and salinity Rayleigh number $R_{\mathrm{S}}$ have stabilizing behavior and the negative range of Soret coefficient $S_{\mathrm{T}}$ and salinity Rayleigh number $R_{\mathrm{S}}$ have destabilizing behavior.

Fig. 3. (a) indicates the variation of $R_{\mathrm{c}}$ versus the salinity Rayleigh number $R_{\mathrm{s}}$ for different values of the ratio of mass transport to the heat transport $\tau$ and $T_{a}=10^{5}$ for oscillatory instability as it is greater than $10^{3}$. When $R_{\mathrm{S}}$ varies from -500 to 500 , the values of $R_{\mathrm{c}}$ decreases and promoting instability. This is imparted to the fluid salted from above and heated from below making the system top heavy. Also if it is salted from below and heated from above the trend is reversed.

In Fig. 3. (b), the variation of $R_{\mathrm{c}}$ versus the Soret parameter $S_{\mathrm{T}}$ for different value of the ratio of mass transport to the heat transport $\tau$ and $T_{a}=10^{5}$ for oscillatory mode. It is clear that the system destabilizes according to increase of $R_{\mathrm{c}}$ and decrease of $S_{\mathrm{T}}$. Also, the cell shape tends to asymptotic trend in Figs. 1. (a) and (b) and Figs. 3. (a) and (b) with respect to horizontal axis.

Fig. 4. (a) shows that the variation of $R_{\mathrm{c}}$ versus $\varepsilon$ for various Taylor number $T_{a}=10$ and 100 . This gives that the system destabilizes because the rotation is so small, when anisotropic parameter $\varepsilon$ increases and $R_{\mathrm{c}}$ decreases.

Fig. 4. (b) analyses the variation of $R_{\mathrm{c}}$ versus $\varepsilon$ for different Taylor numbers $10^{5}$ to $10^{8}$ for oscillatory mode. An increase in Taylor number $T_{a}$ leads to an increase in critical thermal Rayleigh number $R_{\mathrm{c}}$. Clearly, this system tends to stabilize because the entire system of the rotation is much pronounced.

\section{Results and discussion}

The effect of rotation on Soret-driven ferrothermohaline convection saturating an anisotropic porous medium of sparse particle suspension has been investigated. The boundaries are considered to be insulated to temperature and salinity perturbations and the resulting eigen value problem is solved numerically using the Brinkman model as well as analytically employing regular perturbation technique with wave number $a_{c}$ as a perturbation parameter. Also we have investigated the effects of various important parameters on critical wave number and corresponding critical magnetic thermal Rayleigh number on the onset of convection. The principal conclusions of this investigation are as under: 
For the case of stationary convection, anisotropy effect have a destabilizing behavior, whereas rotation have a stabilizing behavior. In the presence of small rotation $\left(T_{a} \leq 10^{2}\right)$, the destabilizing effect of the permeability of the porous medium and anisotropy are depicted in Fig. 1. (a) and Fig. 4. (a). But the presence of rotation is much pronounced $\left(T_{a} \geq 10^{3}\right)$, the permeability of the medium and anisotropy effect may have a destabilizing or a stabilizing effect on the onset of instability. These are depicted in Fig. 1. (b), Figs. 2. (a) and (b), Figs. 3. (a) and (b) and Fig. 4. (b). This is because in the presence of rotation, there is a competition between the destabilizing role of an anisotropy effect and the stabilizing role of rotation.

From the Figures, one can conclude that the magnetization parameters, Soret parameter and rotation have a profound influence on the onset of convection in an anisotropic porous medium. Moreover, anisotropy and Soret effects have a destabilizing behavior and rotation have a stabilizing behavior.

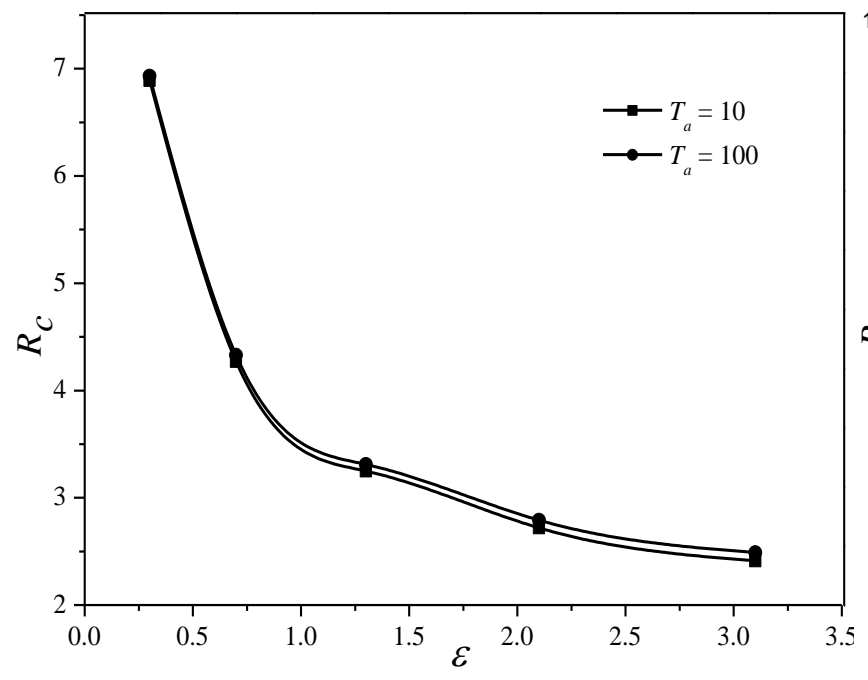

(a)

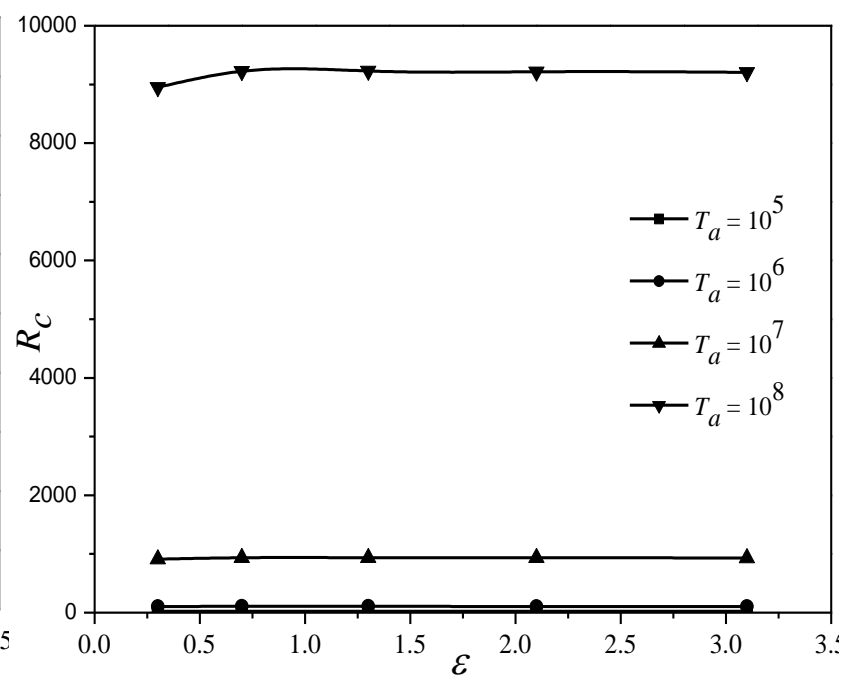

(b)

Fig. 4. (a) Variation of $R_{c}$ versus $\varepsilon$ of stationary instability for $T_{a}=10$ and 100. (b) Variation of $R_{c}$ versus $\varepsilon$ of oscillatory instability for different $T_{a}$.

\section{Acknowledgements}

The authors are grateful to Prof. D. Govindarajulu, Principal, Pondicherry Engineering College, Puducherry, for his constant encouragement. The author K. Raju is thankful to UGC for grant of Rajiv Gandhi National Fellowship 20102011 (Award letter number: F. 14-2(SC)/2010 (SA-III)).

\section{References}

[1] R.E. Rosensweig, Ferrohydrodynamics, Cambridge University Press, Cambridge, (1985).

[2] S. Chandrasekhar, Hydrodynamics and Hydromagnetic stability, Oxford University Press, London, (1961).

[3] B.A. Finlayson, Convective instability of ferromagnetic fluids, International Journal of Fluid Mechanics, Vol.40, (1970), pp.753-767.

[4] D.P. Lalas, S. Carmi, Thermoconvective stability of ferrofluids, Phys. Fluids. Vol.14, (1971), 436-437.

[5] G. Vaidyanathan, R. Sekar, R. Vasanthakumari, Effect of rotation on heat transfer through a strongly magnetized ferrofluids, Presented in the Ninth International Conference on Magnetic fluids at Bremen, Germany during June, (2001), pp.23-27.

[6] A. Mansur, A. Amahmid, M. Hasnaout, M. Mamou, Bifurcation imperfection in double-diffusive-Soret convection in porous reservoirs due to lateral heating, Revue des Energies Renouvelables CER'07 Oujda, (2007), pp.245-248.

[7] R. Sekar, G. Vaidyanathan, Convective instability of a magnetized ferrofluid in a rotating porous medium, International Journal of Engineering Science, Vol.31, (1993), pp.1139-1150.

[8] R. Sekar, G. Vaidyanathan, A. Ramanathan, Ferroconvection in an anisotropic porous medium, International Journal of Engineering Science Vol.34, (1996), pp.399-405.

[9] G. Vaidyanathan, R. Sekar, A. Ramanathan, Effect of rotation and anisotropy of a porous medium on ferroconvection, Indian Journal of Engineering and Materials Sciences Vol.5, (1998), pp. 436-440.

[10] G. Vaidyanathan, R. Sekar, A. Ramanathan, Ferroconvection in an anisotropic densely packed porous medium, Indian Journal of Chemical Technology Vol.9, (2002), pp.446-449.

[11] R. Sekar, G. Vaidyanathan, A. Ramanathan, The ferroconvection in fluids saturating a rotating densely packed porous medium, International Journal of Engineering Science Vol.31, (1993), pp.241-250. 
[12] R. Sekar, G. Vaidyanathan, A. Ramanathan, Effect of magnetic field dependent viscosity on ferroconvection in rotating medium, Indian Journal of Pure and Applied Physics Vol.40, (2002), pp.159-165.

[13] M. Gerth-Noritzsch, D. Yu Borin, S. Odenbach, Anisotropy of the magnetoviscous effect in ferrofluids containing nanoparticles exhibiting magnetic dipole interaction, Journal of Physics Condens Matter. Vol.23, (2011), 346002.

[14] I.S. Shivakumara, Jinho Lee, C.E. Nanjundappa, M. Ravisha, Ferromagnetic convection in a rotating ferrofluid saturated porous layer, Transport in Porous Media Vol.173, (2011), pp.251-273.

[15] Sunil, Divya, Veena Sharma, Effect of dust particles on a rotating ferromagnetic fluid heated from below saturating a porous medium, Journal of Colloid and Interface Science Vol.291, (2005), pp.152-161.

[16] Sunil, Anu Sharma, R. G. Shandil, Effect of rotation on a ferromagnetic fluid heated and soluted from below in the presence of dust particles, Applied Mathematics and Computation Vol.177, (2006), pp.614-628.

[17] Sunil, P. Chand, A. Mahajan, P. Sharma, Effect of rotation on double-diffusive convection in a magnetized ferrofluid with internal angular momentum, Journal of Applied Fluid Mechanics Vol.4, (2011), pp.43-52.

[18] M.S. Malashetty, Ioan Pop, Rajashekhar Heera, Linear and non-linear double diffusive convection in a rotating sparsely packed porous layer using a thermal non-equilibrium model, Continuum Mechanics and Thermodynamics Vol.21, (2009), pp.317-339.

[19] M.S. Malashetty, Rajashekhar Heera, The effect of rotation on the onset of double diffusive convection in an anisotropic porous layer, Transport in Porous Media Vol.74, (2008), pp.105-127.

[20] L.P. Benano-Melly, J.P. Caltagirone, B. Faissat, F. Montel, P. Costesque, Modelling Soret coefficient measurement experiments in porous media considering thermal and solutal convection, International Journal of Heat and Mass Transfer, Vol.44, (2001), pp.1285-1297.

[21] R. Sekar, G. Vaidyanathan, A. Ramanathan, Effect of rotation on ferro thermohaline convection, Journal of Magnetism and Magnetic Material Vol.218, (2000), pp.266-272.

[22] R. Sekar, A. Ramanathan, G. Vaidyanathan, Effect of rotation on ferro thermohaline convection saturating a porous medium, Indian Journal of Engineering and Material Sciences Vol.5, (1998), pp.445-452.

[23] R. Sekar, G. Vaidyanathan, R. Hemalatha, S. Senthilnathan, Effect of coriolis force on a Soret-driven ferrothermohaline convective system, International Journal of Mathematical Science Vol.6, (2007), pp.415-427.

[24] G. Vaidyanathan, R. Sekar, R. Hemalatha, Effect of coriolis force on Soret-driven ferrothermohaline convection in a medium of sparse particle suspension, Indian Journal of Pure and Applied Physics Vol.45, (2007), pp.666-673.

[25] R. Sekar, K. Raju, R. Vasanthakumari, A linear analytical study of Soret-driven ferrothermohaline convection in an anisotropic porous medium, Journal of Magnetism and Magnetic Material Vol.331, (2013), pp.122-128.

[26] P.G. Baines, A.E. Gill, On thermohaline convection with linear gradients, Journal of Fluid Mechanics Vol.37, (1969), pp.289-306. 